OPEN ACCESS

Edited by:

Rabia Johnson,

South African Medical Research

Council, South Africa

Reviewed by:

Celestino Sardu,

University of Campania Luigi Vanvitelli,

Italy

Felice Gragnano,

University of Campania Luigi Vanvitelli,

$$
\text { Italy }
$$

*Correspondence: Yujie Zhou

azzyj12@163.com

Dongmei Shi

18910778615@163.com

${ }^{\dagger}$ These authors have contributed equally to this work and share

last authorship

Specialty section:

This article was submitted to Cardiovascular Endocrinology,

a section of the journal

Frontiers in Endocrinology

Received: 24 December 2021

Accepted: 26 January 2022

Published: 22 February 2022

Citation:

Guo Q, Feng X, Zhang B, Zhai G,

Yang J, Liu Y, Liu Y, Shi D and Zhou Y

(2022) Influence of the Triglyceride-

Glucose Index on Adverse

Cardiovascular and Cerebrovascular

Events in Prediabetic Patients With

Acute Coronary Syndrome.

Front. Endocrinol. 13:843072.

doi: 10.3389/fendo.2022.843072

\section{Influence of the Triglyceride-Glucose Index on Adverse Cardiovascular and Cerebrovascular Events in Prediabetic Patients With Acute Coronary Syndrome}

\author{
Qianyun Guo ${ }^{1}$, Xunxun Feng ${ }^{1}$, Bin Zhang ${ }^{2}$, Guangyao Zhai ${ }^{1}$, Jiaqi Yang ${ }^{1}$, Yang Liu ${ }^{1}$, \\ Yuyang Liu ${ }^{1}$, Dongmei Shi ${ }^{1 * t}$ and Yujie Zhou ${ }^{1 * t}$ \\ ${ }^{1}$ Beijing Key Laboratory of Precision Medicine of Coronary Atherosclerotic Disease, Clinical Center for Coronary Heart \\ Disease, Department of Cardiology, Beijing Anzhen Hospital, Beijing Institute of Heart Lung and Blood Vessel Disease, \\ Capital Medical University, Beijing, China, ${ }^{2}$ Department of Cardiology, Fuwai Hospital, National Center for Cardiovascular \\ Disease, Chinese Academy of Medical Science and Peking Union Medical College, Beijing, China
}

Background: Cardiovascular disease and insulin resistance are closely related. The triglyceride-glucose (TyG) index is frequently used as an indicator of insulin resistance. However, there is scant information on the TyG index in the prediabetic population, nor is the prognostic significance of the index known for prediabetes and acute coronary syndrome (ACS) patients.

Methods: The clinical endpoint was a major adverse cardiovascular and cerebrovascular event (MACCEs), including cardiac-related death, non-fatal myocardial infarction, ischemia-driven revascularization, and stroke. The TyG index was calculated as = In [(triglyceride level, $\mathrm{mg} / \mathrm{dL}$ ) $\times$ (glucose level, $\mathrm{mg} / \mathrm{dL}$ ) $\div 2$ ] under fasting conditions.

Results: The study included 2,030 prediabetic patients with ACS. Patients were followed up for 2.5 years, during which the total incidence of MACCEs was $12 \%$. After adjustment for covariates, the TyG index was found to be predictive of prediabetes with ACS (HR 4.942, 95\% Cl: 3.432-6.115, $\mathrm{P}<0.001)$. Using propensity score matching, 574 pairs were successfully matched, and the two groups were analyzed in terms of survival. This showed that there was a significantly greater incidence of MACCEs in patients with high TyG indices (HR 3.526, 95\%Cl: 2.618-4.749, $\mathrm{P}<0.001$ ), mainly due to ischemia-driven revascularization and stroke.

Conclusions: The TyG index independently predicts future MACCEs and may be an important prognostic indicator for patients with prediabetes and ACS.

Keywords: TyG index, prediabetes, ACS, prognosis, MACCEs 


\section{BACKGROUND}

The prevalence of diabetes has risen from 108 million to 422 million in the last thirty years, of which type 2 diabetes (T2DM) accounts for more than $90 \%$, and studies estimate that it will increase to 642 million by 2040 . Most patients go through a prediabetic stage before they develop diabetes $(1,2)$. The prevalence of prediabetes is also rising globally. Research shows that by 2030, more than 470 million people will suffer from prediabetes. Prediabetes is associated with co-existing insulin resistance (IR) and $\beta$-cell dysfunction, and these abnormalities begin before blood sugar changes are detected. Prediabetes is a complex, multi-factorial metabolic disorder, and its pathophysiology centers around IR, impaired incretin action, and high insulin secretion. Observational evidence has linked prediabetes with an elevated risk of nephropathy, diabetic retinopathy, and cardiovascular diseases (CVDs) (3-5).

IR is usually present in prediabetes, before development to T2DM (6). It has been found that the triglyceride-glucose (TyG) index is a practical method of evaluating IR, and the TyG index has been verified in multiple populations around the world, and its effectiveness and reliability was consistently showed in IR detecting $(7,8)$. Recent studies suggest that prediabetes is correlated with an increased risk of T2DM, CVD, dementia, and cancer; moreover, its incidence is increasing worldwide (9). In China, the prevalence of prediabetes rose from $15.5 \%$ in 2008 to $35.2 \%$ in 2017, with about 357 million people suffering from prediabetes $(10,11)$. Notably, the best control of glycemia and IR as in the case of metformin therapy could result in these patients in best clinical outcomes $(12,13)$. Moreover, asymptomatic diabetic patients with high TyG indices have a higher risk of coronary artery stenosis (14). Therefore, the TyG index may be a valuable biomarker for the development of diabetes $(15,16)$, allowing the effective screening and early detection of individuals at high risk for T2DM (17). In addition, it has been found that after adjustment for confounders, there was a close relationship between the TyG index and prediabetes (18).

In addition, the TyG index, which also measures IR, may be helpful for the early recognition of cardiovascular events, with higher TyG indices in high-risk groups related to an increased risk of CVD (19). Although there has been an increase in the

Abbreviations: T2DM, type 2 diabetes; IR, insulin resistance; CVDs, cardiovascular diseases; TyG, triglyceride-glucose; ACS, acute coronary syndrome; MACCEs, major adverse cardiovascular and cerebrovascular events; HbAlc, glycosylated hemoglobin Alc; UAP, unstable angina pectoris; NSTEMI, non-ST-segment elevation myocardial infarction; STEMI, ST-segment elevation myocardial infarction; CABG, coronary artery bypass grafting; ROC, receiver operating characteristic; BMI, body mass index; SBP, systolic blood pressure; DBP, diastolic blood pressure; TG, triglyceride; FBG, fasting blood glucose; TC, total cholesterol; LDL-C, low-density lipoprotein cholesterol; HDL-C, high density lipoprotein cholesterol; Cr, creatinine; SUA, serum uric acid; CRP, C-reactive protein; hs-TNI, high sensitivity troponin I; MI, myocardial infarction; IQR, interquartile range; HRs, hazard ratios; RCS, restricted cubic spline; $\mathrm{CI}$, confidence level; ACEI, angiotensin-converting enzyme inhibitor; AUC, area under curve; $\mathrm{BNP}$, brain natriuretic peptide; eGFR, estimated glomerular filtration rate; HOMA-IR, Homeostasis model assessment of insulin resistance; CAC, coronary artery calcification; CAD, coronary artery disease; CTA, computed tomography angioplasty; ICU, intensive care unit; PCI, percutaneous coronary intervention; AMI, acute myocardial infarction. number of studies on the TyG index and CVDs recently, there is still a lack of prognosis-related studies on acute coronary syndrome (ACS) in prediabetic patients. Identification of an effective means of evaluating the prognosis of prediabetic patients with ACS would assist the recognition of those at high risk of major adverse cardiovascular and cerebrovascular events (MACCEs) for closer monitoring or potential early intervention. Based on the results of follow-up, we aimed to explore the relationship between the prognosis and the TyG index in prediabetic patients with ACS.

\section{METHODS}

\section{Study Design, Patient Population and Definitions}

This single center, retrospective, observational study enrolled 2030 patients with prediabetes and ACS admitted to Anzhen Hospital for coronary angiography from August 2018 to September 2019. Using the definition of prediabetes, we discussed and reviewed previous studies and found that although the new glycosylated hemoglobin Alc (HbA1c) criteria identified fewer high-risk individuals than those with impaired fasting glucose, HbAlc (in the range of 5.7-6.4\%) had a similar the predictive value to impaired fasting glucose alone (20). At the same time, considering that the fasting glucose of patients admitted to hospital may be affected by their diet, the final definition was based on the 2021 AHA Classification and Diagnosis of Diabetes, with an $\mathrm{HbAlc}$ range from $5.7 \%$ to $6.4 \%$ (21). ACS was defined based on appropriate guidelines, including unstable angina pectoris (UAP), ST-segment elevation myocardial infarction (STEMI) and non-ST-segment elevation myocardial infarction (NSTEMI) (22). The exclusion criteria were patients with (1) abnormal liver function: severe insufficiency with alanine transaminase (ALT) or aspartate transaminase (AST) over 5 upper limit of normal; abnormal kidney function: with estimated glomerular filtration rate (eGFR) $<30 \mathrm{~mL} /\left(\mathrm{min}{ }^{*} 1.73 \mathrm{~m}^{2}\right)$; (2) incomplete baseline and follow-up clinical data; (3) a history of coronary artery bypass grafting (CABG); (4) any kind of cancer or other major diseases affecting long-term survival. The TyG index cut-off was calculated using the receiver operating characteristic (ROC) curve (TyG index = 8.83). The patients were assigned to two groups based on their TyG indices, the "high TyG index group" (TyG index $\geq 8.83$ ) and the "low TyG index group" (TyG index $<8.83$ ). Patients were matched through the 1:1 propensity score between the two groups, and 574 pairs were successfully identified for survival analysis. These details are shown in the flow chart (Figures 1 and 2).

\section{Data Collection and Laboratory Examination}

We performed the data collection of clinical characteristics using case report form, including age, gender, body mass index (BMI), systolic blood pressure (SBP), diastolic blood pressure (DBP), previous medical history, laboratory examination and types of medication taken. The TyG index was defined as previously reported: TyG index $=\ln$ (fasting triglyceride $(\mathrm{TG}, \mathrm{mg} / \mathrm{dL}) \times$ 


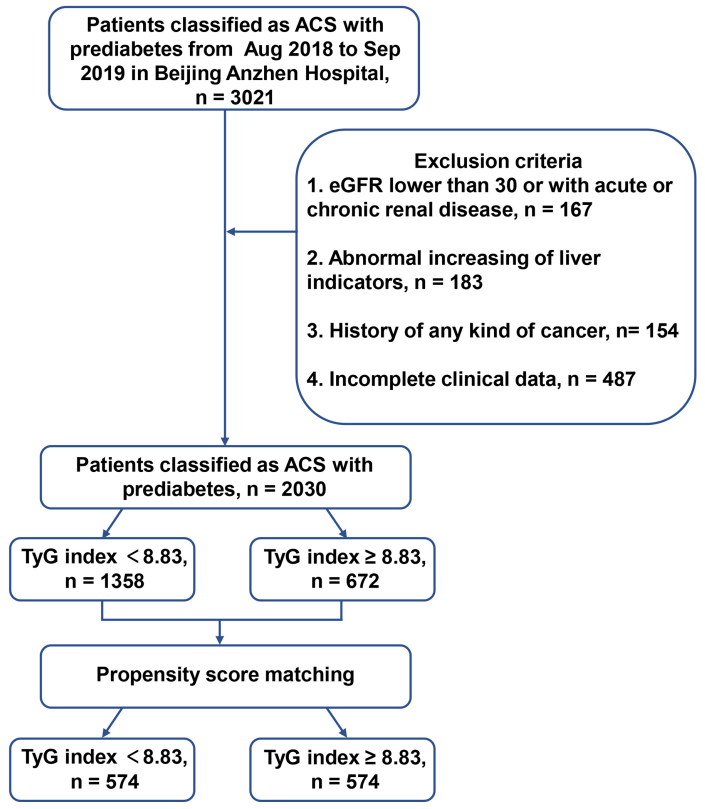

FIGURE 1 | The flow chart of enrolled patients. ACS, acute coronary syndrome; HbA1c, glycosylated hemoglobin A1c; DM, diabetes mellitus; eGFR, estimated glomerular filtration rate; TyG, triglyceride-glucose.

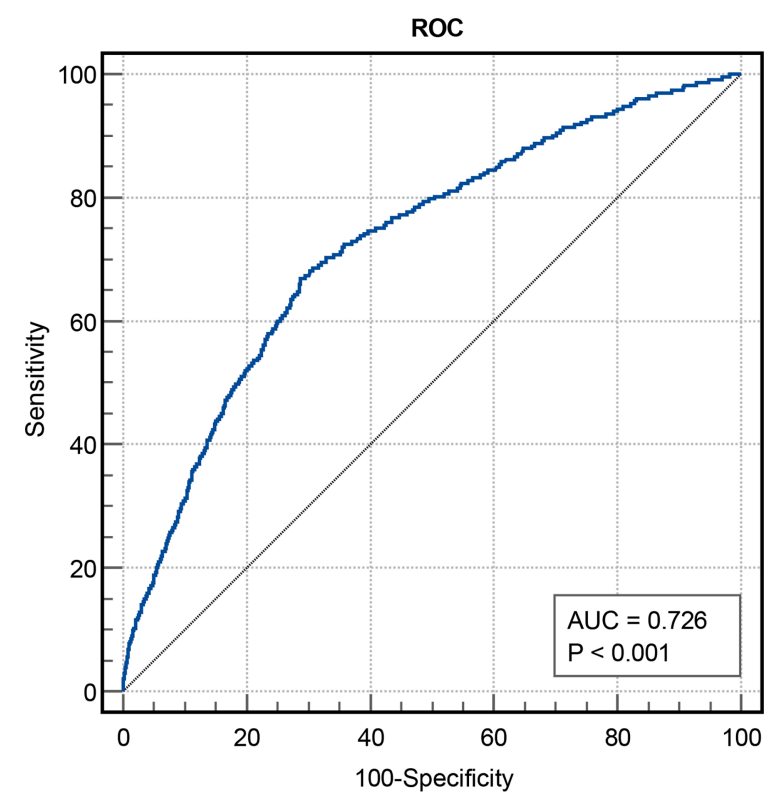

FIGURE 2 | The predictive values of TyG index for the risk of MACCEs. TyG, triglyceride-glucose; MACCEs, major adverse cardiovascular and cerebrovascular events; AUC, area under curve.

fasting blood glucose level (FBG, mg/dL)/2) (23). The collection of peripheral venous blood samples was performed in the morning after an overnight fast and immediately sent for analysis to the central laboratory methods of Beijing Anzhen
Hospital. The analysis included TG, total cholesterol (TC), lowdensity lipoprotein cholesterol (LDL-C), high density lipoprotein cholesterol (HDL-C), creatinine (Cr), serum uric acid (SUA), FBG, HbA1c, C-reactive protein (CRP), high sensitivity troponin I (hs-TNI), amongst other hematological and biochemical parameters. All of the above biochemical variables were evaluated at baseline.

\section{Definition of Clinical Endpoints}

All patients were routinely followed up at 3, 6 and 12 months, and then annually for 30 months. Information on adverse events was gained from patients or their families by telephone questionnaires. New-onset MACCEs was defined as the primary endpoint comprising cardiac-related death, non-fatal myocardial infarction (MI), ischemia-driven revascularization, and stroke. Secondary endpoints included the same events $(24,25)$, which were recorded for each patient during the 2.5-year follow-up after discharge. The first primary endpoint event that occurred during the follow-up was used for analysis. For multiple adverse outcomes during the follow-up, only the most severe event was used (cardiac death $>\mathrm{MI} /$ stroke $>$ ischemia-driven revascularization).

\section{Statistical Analysis}

Normally distributed continuous variables were represented as means \pm standard deviation, while those with skewed distribution were shown as median (interquartile range [IQR]). Besides, the continuous variables between two groups were compared using one-way analysis of variance or the KruskalWallis test, and the categorical variables were showed as frequencies and analyzed using test of chi-square. The Pearson and Spearman correlation analyses were applied to assess the relationship between two variables as appropriate. Time dependent Cox proportional hazards regression was performed to evaluate the hazard ratios (HRs) for MACCEs associated with the predictive values of TyG and variables. In multivariate analyses, in addition to age, gender (reference to male), BMI, SBP, DBP, smoking status, history of hypertension and hyperlipemia, levels of LDL-C, HDL-C, Cr, SUA, eGFR, brain natriuretic peptide (BNP), and CRP were adjusted. Restricted cubic spline (RCS) regression was used to show the graphical association between TyG index and MACCEs. ROC curves were used to assess the cut-off point of the TyG index for MACCEs. To match the patients between the high-TyG index and low-TyG index groups, a 1:1 propensity score-matched analysis was conducted (details in Supplementary Material). Kaplan-Meier analysis was performed to evaluate the time-related events and the evaluation of discrepancies was assessed by log-rank tests. Two-tailed P-values $<0.05$ were regarded as statistically significant. All statistical analyses in this study used MedCalc version 20.0.3, SPSS version 22.0 and $\mathrm{R}$ version 4.0.0.

\section{RESULTS}

\section{Baseline Characteristics}

In total, 2030 patients were enrolled in the study. During 2.5 years of follow-up, 233 (11.5\%) of the 2030 patients experienced 
MACCEs, including $11(0.5 \%)$ cardiac deaths, 29 (1.4\%) nonfatal MIs, 180 (8.9\%) ischemia-driven revascularizations and 41 (2.0\%) strokes. Using stratification based on MACCEs occurrence, the baseline clinical characteristics of the overall population were grouped into MACCEs and non-MACCEs (Table 1). The TyG index levels in the MACCEs group (9.00 \pm $0.51)$ were significantly elevated compared to those in the nonMACCEs group $(8.59 \pm 0.48)(\mathrm{P}<0.001)$. The two groups also differed significantly in terms of TC, TG, HDL-C, Cr, SUA, FBG, HbAlc, hs-TNI, and use of angiotensin-converting enzyme inhibitor $(\mathrm{ACEI})$ medication $(\mathrm{P}<0.05)$ but not in terms of other indicators. The area under the curve (AUC) of the TyG index for MACCEs was 0.726 (95\% CI 0.691-0.761, P < 0.001, $\mathrm{P}<0.001)$. The TyG index of 8.83 was determined as the optimal cutoff point for predicting MACCEs with a sensitivity of $64.4 \%$ and a specificity of $71.7 \%$. Patients with raised TyG indices tended seemed to be younger, with high BMI and DBP levels and a greater incidence of hypertension compared to the lower TyG index group. Besides these, the levels of LDL-C, TC, TG, Cr, SUA, FBG, and $\beta$-blocker use were higher in the group with high TyG indices $(\mathrm{P}<0.01)$, while the HDL-C levels were lower in the group with low TyG indices $(\mathrm{P}<0.001)$ (Table 2$)$.

\section{The TyG Index and Indicators of Cardiovascular Risk}

The correlations between traditional cardiovascular risk indicators or commonly-used risk indicators of CVD and the TyG index were examined. The TyG index was positively linked to DBP, history of hyperlipemia, and smoking status $(\mathrm{P}<0.05)$ while correlating negatively with gender, HDL-C and BNP $(\mathrm{P}<0.05)$ (Figure 3).

\section{The TyG Index and MACCEs}

After the stratification of the MACCEs incidence, propensity score matching was performed (Figure 4). This showed

TABLE 1 | Baseline clinical characteristics among the MACCEs and non-MACCEs group of overall population.

\begin{tabular}{|c|c|c|c|c|}
\hline Characteristics & Overall $(n=2030)$ & Non-MACCEs $(n=1797)$ & MACCEs $(n=233)$ & $\mathbf{P}$ \\
\hline \multicolumn{5}{|l|}{ Demographic } \\
\hline Age, years & $58.87 \pm 10.27$ & $59.02 \pm 10.29$ & $57.74 \pm 10.06$ & 0.074 \\
\hline Gender, (male\%) & $1505(74.1)$ & $1327(73.8)$ & $178(76.4)$ & 0.449 \\
\hline BMI, $\mathrm{kg} / \mathrm{m}^{2}$ & $25.64 \pm 3.31$ & $25.59 \pm 3.34$ & $25.97 \pm 3.08$ & 0.107 \\
\hline SBP, mmHg & $128.42 \pm 16.83$ & $128.46 \pm 16.84$ & $128.06 \pm 16.77$ & 0.735 \\
\hline $\mathrm{DBP}, \mathrm{mmHg}$ & $76.91 \pm 10.86$ & $76.86 \pm 10.76$ & $77.26 \pm 11.63$ & 0.603 \\
\hline \multicolumn{5}{|l|}{ Medical history, n (\%) } \\
\hline Smoking & $1026(50.5)$ & 902 (50.2) & $124(53.2)$ & 0.424 \\
\hline Hypertension & $1214(59.8)$ & $1074(59.8)$ & $140(60.1)$ & 0.982 \\
\hline Hyperlipemia & $1412(69.6)$ & $1252(69.7)$ & $160(68.7)$ & 0.813 \\
\hline Pre-PCI & 479 (23.6) & $425(23.7)$ & $54(23.2)$ & 0.937 \\
\hline \multicolumn{5}{|l|}{ Laboratory results } \\
\hline LDL-C, mmol/L & $2.41 \pm 0.84$ & $2.40 \pm 0.85$ & $2.46 \pm 0.84$ & 0.323 \\
\hline $\mathrm{TC}, \mathrm{mmol} / \mathrm{L}$ & $4.04 \pm 0.98$ & $4.03 \pm 0.98$ & $4.16 \pm 1.02$ & 0.046 \\
\hline $\mathrm{TG}, \mathrm{mmol} / \mathrm{L}$ & $1.38 \pm 0.71$ & $1.32 \pm 0.64$ & $1.86 \pm 0.96$ & $<0.001$ \\
\hline HDL-C, mmol/L & $1.14 \pm 0.26$ & $1.15 \pm 0.27$ & $1.07 \pm 0.22$ & $<0.001$ \\
\hline TyG index & $8.64 \pm 0.50$ & $8.59 \pm 0.48$ & $9.00 \pm 0.51$ & $<0.001$ \\
\hline $\mathrm{Cr}, \mu \mathrm{mol} / \mathrm{L}$ & $70.86 \pm 14.80$ & $70.61 \pm 14.54$ & $72.82 \pm 16.58$ & 0.032 \\
\hline SUA, $\mu \mathrm{mol} / \mathrm{L}$ & $355.37 \pm 88.15$ & $352.97 \pm 85.75$ & $373.86 \pm 103.20$ & 0.001 \\
\hline eGFR, $\mathrm{mL} /\left(\min ^{*} 1.73 \mathrm{~m}^{2}\right)$ & $96.48 \pm 13.09$ & $96.50 \pm 12.98$ & $96.34 \pm 13.94$ & 0.858 \\
\hline BNP, pg/mL & $47.33 \pm 102.55$ & $47.35 \pm 101.94$ & $47.16 \pm 107.36$ & 0.979 \\
\hline CRP, mg/L & $3.17 \pm 5.56$ & $3.13 \pm 5.55$ & $3.48 \pm 5.63$ & 0.369 \\
\hline FBG, $\mathrm{mmol} / \mathrm{L}$ & $5.83 \pm 1.26$ & $5.77 \pm 1.21$ & $6.25 \pm 1.56$ & $<0.001$ \\
\hline HbA1c, \% & $6.00 \pm 0.24$ & $5.99 \pm 0.24$ & $6.05 \pm 0.24$ & 0.002 \\
\hline hs-TNI, pg/mL & $0.36 \pm 2.42$ & $0.30 \pm 2.12$ & $0.80 \pm 4.02$ & 0.003 \\
\hline \multicolumn{5}{|l|}{ Clinical presentation, $\mathrm{n}(\%)$} \\
\hline STEMI & $118(5.8)$ & $98(5.5)$ & $20(8.6)$ & 0.076 \\
\hline NSTEMI & $108(5.3)$ & $94(5.2)$ & $14(6.0)$ & 0.732 \\
\hline UAP & $1804(88.9)$ & 1605 (89.3) & $199(85.4)$ & 0.094 \\
\hline \multicolumn{5}{|l|}{ Medication, n (\%) } \\
\hline Antiplatelet & 2029 (100.0) & 1796 (99.9) & $233(100.0)$ & 1.000 \\
\hline Statin & 2026 (99.8) & $1793(99.8)$ & $233(100.0)$ & 1.000 \\
\hline ACEI & $337(16.6)$ & $284(15.8)$ & $53(22.7)$ & 0.010 \\
\hline ARB & $1504(74.1)$ & $1320(73.5)$ & $184(79.0)$ & 0.084 \\
\hline$\beta$-blocker & 1608 (79.2) & $1422(79.1)$ & $186(79.8)$ & 0.872 \\
\hline Nitrate & $1841(90.7)$ & $1628(90.6)$ & $213(91.4)$ & 0.775 \\
\hline
\end{tabular}

BMI, body mass index; SBP, systolic blood pressure; DBP, diastolic blood pressure; PCI, percutaneous coronary intervention; LDL-C, low-density lipoprotein cholesterol; TC, total cholesterol; TG, triglyceride; HDL-C, high density lipoprotein cholesterol; TyG, triglyceride-glucose; Cr, creatinine; SUA, serum uric acid; eGFR, estimated glomerular filtration rate; BNP, brain natriuretic peptide; CRP, C-reactive protein; FBG, fasting blood glucose; HbA1c, glycosylated hemoglobin A1c; hs-TNI, high sensitivity troponin I; STEMI, ST-segment elevation myocardial infarction; NSTEMI, non-ST-segment elevation myocardial infarction; UAP, unstable angina pectoris; ACEl, angiotensin-converting enzyme inhibitor; ARB, angiotensin receptor blocker. 
TABLE 2 | Baseline clinical characteristics of patients stratified by the optimal cutoff point of TyG index.

\begin{tabular}{|c|c|c|c|c|}
\hline Characteristics & Overall $(n=2030)$ & Lower TyG index $(<8.83, n=1358)$ & Higher TyG index $(\geq 8.83, n=672)$ & $\mathbf{P}$ \\
\hline \multicolumn{5}{|l|}{ Demographic } \\
\hline Age, years & $58.87 \pm 10.27$ & $59.70 \pm 10.17$ & $57.21 \pm 10.28$ & $<0.001$ \\
\hline Gender, (male\%) & $1505(74.1)$ & $1021(75.2)$ & $484(72.0)$ & 0.140 \\
\hline BMI, $\mathrm{kg} / \mathrm{m}^{2}$ & $25.64 \pm 3.31$ & $25.39 \pm 3.35$ & $26.14 \pm 3.18$ & $<0.001$ \\
\hline SBP, mmHg & $128.42 \pm 16.83$ & $128.45 \pm 17.02$ & $128.35 \pm 16.45$ & 0.907 \\
\hline DBP, mmHg & $76.91 \pm 10.86$ & $76.52 \pm 10.92$ & $77.69 \pm 10.72$ & 0.022 \\
\hline \multicolumn{5}{|l|}{ Medical history, n (\%) } \\
\hline Smoking & $1026(50.5)$ & $689(50.7)$ & $337(50.1)$ & 0.840 \\
\hline Hypertension & $1214(59.8)$ & $791(58.2)$ & $423(62.9)$ & 0.047 \\
\hline Hyperlipemia & $1412(69.6)$ & $939(69.1)$ & $473(70.4)$ & 0.603 \\
\hline Pre-PCI & 479 (23.6) & $337(24.8)$ & $142(21.1)$ & 0.074 \\
\hline \multicolumn{5}{|l|}{ Laboratory results } \\
\hline LDL-C, mmol/L & $2.41 \pm 0.84$ & $2.30 \pm 0.82$ & $2.63 \pm 0.85$ & $<0.001$ \\
\hline TC, mmol/L & $4.04 \pm 0.98$ & $3.88 \pm 0.93$ & $4.37 \pm 1.00$ & $<0.001$ \\
\hline TG, mmol/L & $1.38 \pm 0.71$ & $1.05 \pm 0.32$ & $2.05 \pm 0.79$ & $<0.001$ \\
\hline HDL-C, mmol/L & $1.14 \pm 0.26$ & $1.18 \pm 0.27$ & $1.06 \pm 0.23$ & $<0.001$ \\
\hline TyG index & $8.64 \pm 0.50$ & $8.37 \pm 0.33$ & $9.17 \pm 0.31$ & $<0.001$ \\
\hline $\mathrm{Cr}, \mu \mathrm{mol} / \mathrm{L}$ & $70.86 \pm 14.80$ & $70.24 \pm 14.10$ & $72.12 \pm 16.05$ & 0.007 \\
\hline SUA, $\mu \mathrm{mol} / \mathrm{L}$ & $355.37 \pm 88.15$ & $345.40 \pm 82.50$ & $375.50 \pm 95.54$ & $<0.001$ \\
\hline eGFR, $\mathrm{mL} /\left(\mathrm{min}^{\star} 1.73 \mathrm{~m}^{2}\right)$ & $96.48 \pm 13.09$ & $96.55 \pm 12.50$ & $96.35 \pm 14.21$ & 0.742 \\
\hline BNP, pg/mL & $47.33 \pm 102.55$ & $49.61 \pm 104.01$ & $42.72 \pm 99.47$ & 0.155 \\
\hline CRP, mg/L & $3.17 \pm 5.56$ & $3.10 \pm 5.66$ & $3.31 \pm 5.36$ & 0.419 \\
\hline FBG, mmol/L & $5.83 \pm 1.26$ & $5.54 \pm 0.97$ & $6.40 \pm 1.56$ & $<0.001$ \\
\hline HbA1c, \% & $6.00 \pm 0.24$ & $6.00 \pm 0.24$ & $6.00 \pm 0.24$ & 0.763 \\
\hline hs-TNI, pg/mL & $0.36 \pm 2.42$ & $0.31 \pm 2.40$ & $0.45 \pm 2.46$ & 0.215 \\
\hline \multicolumn{5}{|l|}{ Clinical presentation, $\mathbf{n}(\%)$} \\
\hline STEMI & $118(5.8)$ & $79(5.8)$ & $39(5.8)$ & 0.999 \\
\hline NSTEMI & 108 (5.3) & $68(5.0)$ & $40(6.0)$ & 0.431 \\
\hline UAP & 1804 (88.9) & $1211(89.2)$ & $593(88.2)$ & 0.580 \\
\hline \multicolumn{5}{|l|}{ Medication, n (\%) } \\
\hline Antiplatelet & 2029 (100.0) & 1358 (100.0) & $671(99.9)$ & 0.719 \\
\hline Statin & 2026 (99.8) & 1354 (99.7) & $672(100.0)$ & 0.381 \\
\hline ACEI & 337 (16.6) & $212(15.6)$ & 125 (18.6) & 0.101 \\
\hline ARB & $1504(74.1)$ & $1020(75.1)$ & $484(72.0)$ & 0.150 \\
\hline$\beta$-blocker & $1608(79.2)$ & $1049(77.2)$ & 559 (83.2) & 0.002 \\
\hline Nitrate & 1841 (90.7) & 1235 (90.9) & 606 (90.2) & 0.634 \\
\hline
\end{tabular}

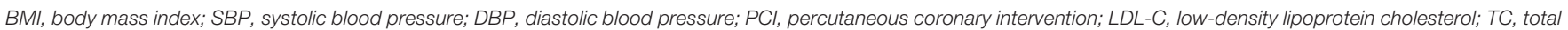
cholesterol; TG, triglyceride; HDL-C, high density lipoprotein cholesterol; TyG, triglyceride-glucose; Cr, creatinine; SUA, serum uric acid; eGFR, estimated glomerular filtration rate;

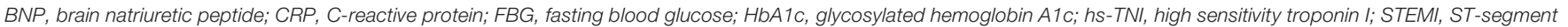
elevation myocardial infarction; NSTEMI, non-ST-segment elevation myocardial infarction; UAP, unstable angina pectoris; ACEl, angiotensin-converting enzyme inhibitor; ARB, angiotensin receptor blocker.

significant differences between the low and high-TyG index groups in terms of gender, age, SBP, DBP, BMI, statin therapy, TC, SUA, LDL-C, Cr, BNP, CRP, eGFR, history of hyperlipemia, hypertension and smoking was not found.

\section{Prediction of MACCEs Using the TyG Index}

Among the overall population, in the univariate Cox proportional hazard analysis, the TyG index, Cr, and SUA were found to be independently related with MACCEs $(\mathrm{P}<0.05)$. In the multivariate analysis, the variables gender, age, BMI, LDL-C, HDL-C, SBP, DBP, TyG index, Cr, SUA, eGFR, $\mathrm{BNP}, \mathrm{CRP}$, history of smoking, hypertension, and hyperlipemia were adjusted. It was found that after adjustment, only the TyG index was independently related to MACCEs (Table 3). Using RCS, we evaluated the shape of the association using penalized splines to examine the relationship between MACCEs risk and the TyG index. In populations of pre/post propensity score matching, with increased TyG index, the RCS curves showed the same trend of monotonic increase in the risk of MACCEs (Figure 5).

\section{The TyG Index and Clinical Outcomes}

The occurrence of MACCEs, ischemia-driven revascularization, and stroke differed significantly between the groups $(\mathrm{P}<0.01)$, shown by pre/post propensity score matching and Cox proportional hazard analyses (Table 4). Kaplan-Meier survival analysis confirmed a greater incidence of MACCEs $(\mathrm{P}<0.001)$, ischemia-driven revascularization $(\mathrm{P}<0.001)$, and stroke $(\mathrm{P}=$ 0.002) in the higher TyG index group (Figure 6).

\section{DISCUSSION}

This appears to be the first investigation of the relationship between prognosis and the TyG index in prediabetic patients with ACS. Firstly, the study included 2030 patients with 


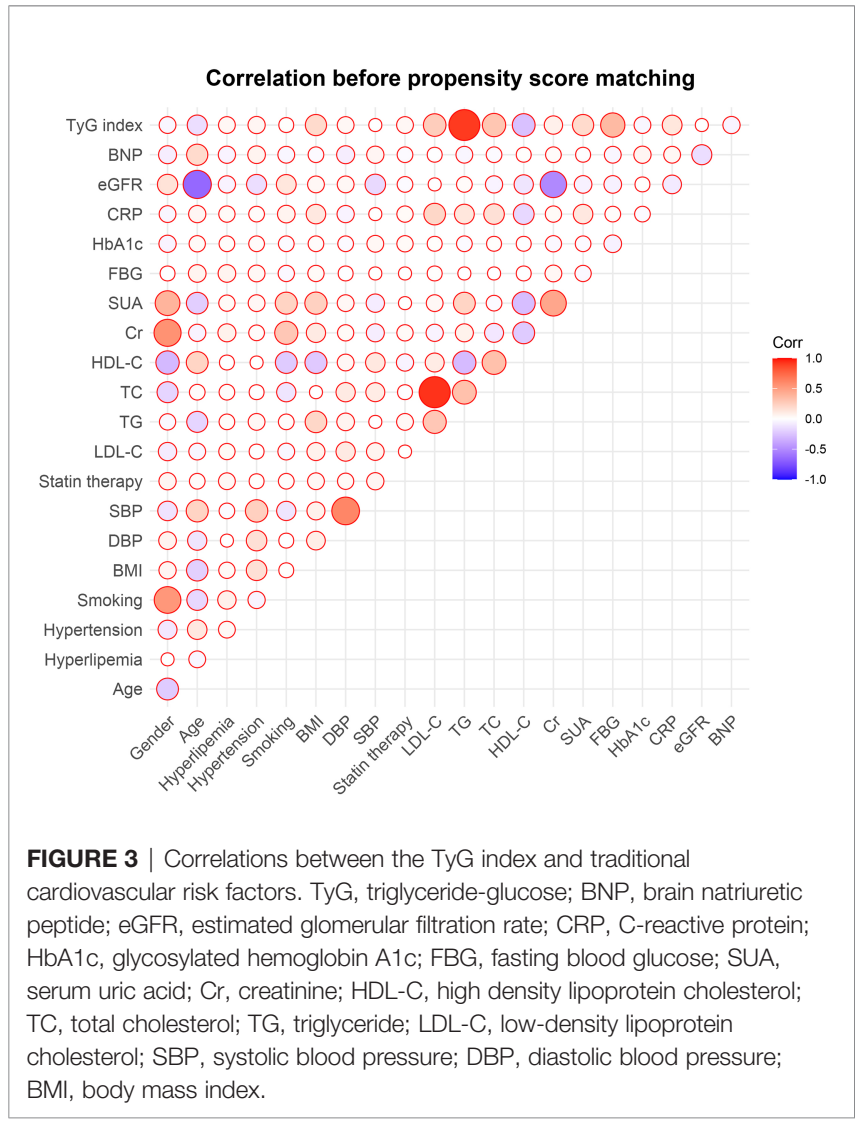

prediabetes and ACS who were followed up for two-and-a-half years during which the total incidence of MACCEs was $11.5 \%$. After 1:1 propensity score matching, MACCEs incidence to be significantly greater in patients with high TyG indices, with ischemia-driven revascularization and stroke dominating. Secondly, multivariate Cox regression analysis of the overall population showed a significant link between the TyG index and MACCEs, and the RCS curve also indicated a consistent increase between the index and the HR both before and after matching.

\section{The TyG Index and Atherosclerosis}

The TyG index has long been regarded as an IR indicator. IR in the liver is frequently estimated by the homeostasis model assessment of insulin resistance (HOMA-IR). Kim et al. concluded that TyG index was more effective HOMA-IR in predicting coronary artery calcification (CAC) (26), and Park et al. have also pointed out that the TyG index predicts CAC progression, especially in adults with non-severe CAC (27). IR has also been found to influence plaque development by promoting apoptosis in vascular smooth muscle cells, macrophages and endothelial cells (28). In addition, studies have shown that prediabetic patients have more severe coronary atherosclerosis and plaque vulnerability than nondiabetic patients (29). Considering the substantial impact of diabetes on CAC, these results may be related to the increased prevalence of diabetes and the increase in the TyG index (30),

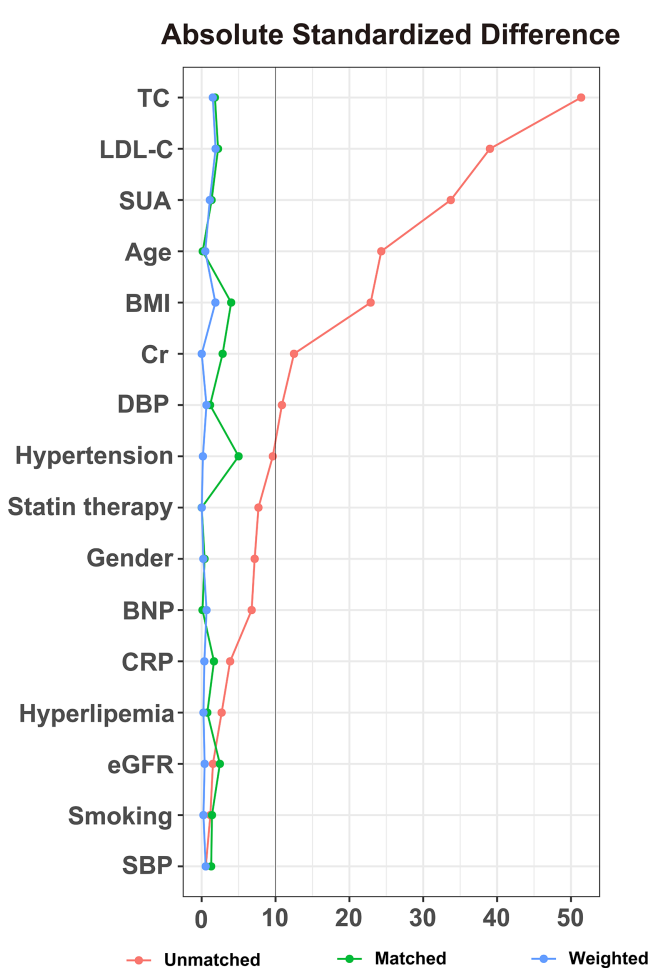

FIGURE 4 | Absolute standardized differences in unweighted and propensity score-weighted data sensitivity analyses. TC, total cholesterol; LDL-C, lowdensity lipoprotein cholesterol; SUA, serum uric acid; BMI, body mass index; $\mathrm{Cr}$, creatinine; DBP, diastolic blood pressure; BNP, brain natriuretic peptide; CRP, C-reactive protein; eGFR, estimated glomerular filtration rate; SBP, systolic blood pressure.

where the critical mechanisms may include the activation of endothelial dysfunction, vascular inflammation, and oxidative stress by hyperglycemic injury (31). An observational study reported that in asymptomatic diabetic patients, the CAC score can be used effectively to assess obstructive coronary artery disease (CAD) by coronary computed tomography angioplasty (CTA) (32). Subsequently, observing coronary plaques through CTA, Won et al. also found a correlation between the TyG index and severity of $\mathrm{CAD}$, suggesting that IR accompanied by CAC affects the progression of coronary plaques (33).

\section{Prediction of MACCEs by the TyG Index in Prediabetic Patients The TyG Index and Stroke}

An association between the TyG index and increased probability of IR recurrence, neurological decline, and all-cause mortality has been found in patients with ischemic stroke (34). A higher TyG index can also predict a poor functional prognosis of acute ischemic stroke (35). In addition, the TyG index is able to forecast both the hospitalization and intensive care unit mortality after stroke, especially with ischemic stroke (36). Shi et al. considered that TyG could assist in the assessment of the probability of ischemic stroke (37). In addition, an increased 
TABLE 3 | Independent predictors of MACCEs in overall patients.

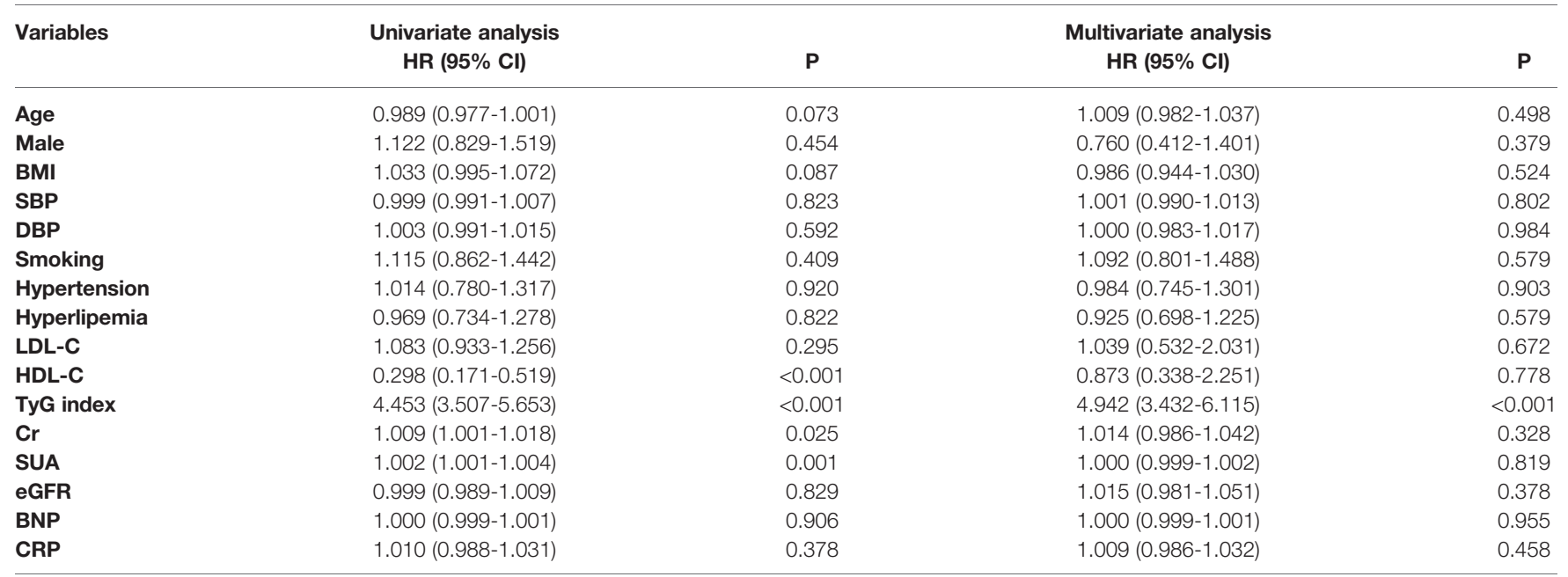

HR, hazard ratio; Cl, confidence level; BMI, body mass index; SBP, systolic blood pressure; DBP, diastolic blood pressure; LDL-C, low-density lipoprotein cholesterol; HDL-C, high density lipoprotein cholesterol; TyG, triglyceride-glucose; Cr, creatinine; SUA, serum uric acid; eGFR, estimated glomerular filtration rate; BNP, brain natriuretic peptide; CRP, C-reactive protein.
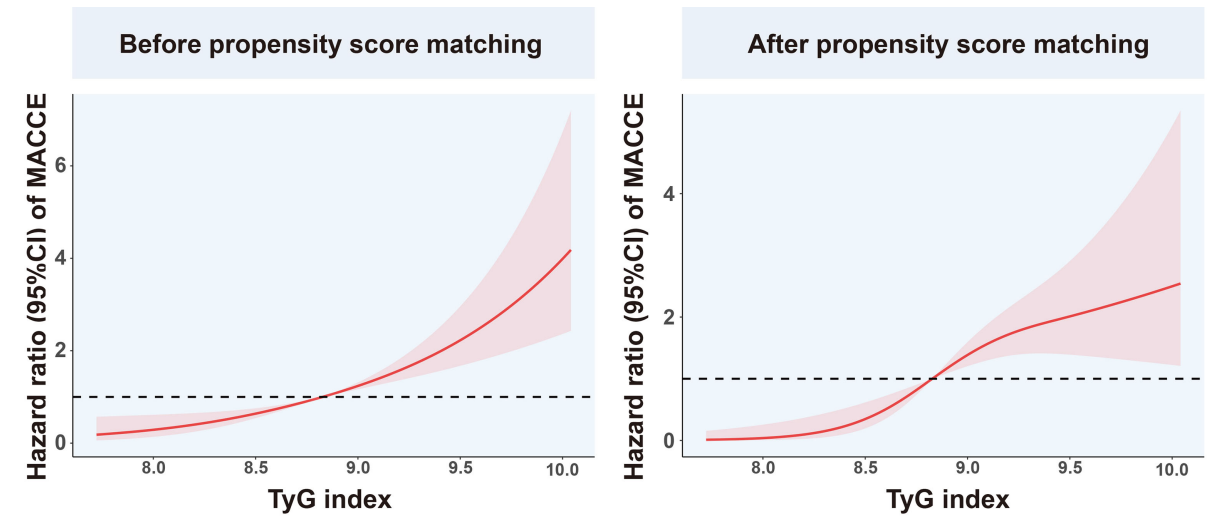

FIGURE 5 | Restricted cubic spline analysis of the association between TyG index and MACCEs. TyG, triglyceride-glucose; MACCEs, major adverse cardiovascular and cerebrovascular events.

TyG index independently predicts the risk of ischemic stroke in the general population, and IR may be positively correlated with future stroke risk (38). Although studies have found that the correlation between BMI and stroke prognosis is not affected by the TyG index (39), it is interesting that Du et al. reported an association between the TyG-BMI index derived from the TyG index and ischemic stroke (40).

A recent registration study observed a relationship between elevated higher TyG indices and increased MACCEs risk with STEMI, and concluded that the TyG index can effectively predict outcomes in STEMI cases after percutaneous coronary intervention (PCI) (41). Subsequently, Zhang et al., in examining the relationship between the TyG index and MACCEs, concluded that the former was valuable in assessing both prognosis and risk stratification in T2DM patients after suffering acute myocardial infarction (AMI) (42). In our research, we found a $2.0 \%$ incidence of stroke in all patients during the 2.5-year follow-up. After propensity score matching, survival analysis showed that the risk of stroke and the TyG index were significantly different between higher and lower TyG groups, which is consistent with previous studies. In addition, the higher TyG index group had a poorer prognosis (HR 2.902, 95\% CI: 1.431-5.885, $\mathrm{P}<0.001$ ), and it was shown that the TyG index may be closely linked to the occurrence of stroke in prediabetic patients with ACS.

\section{The TyG Index and Cardiovascular Events}

Evidence shows that the TyG index is predictive of cardiovascular events. Perusal of the recent literature shows that there is similar evidence in different populations of CAD. Firstly, in SAP patients, the TyG index correlated positively with cardiovascular events, indicating that TyG is a valuable indicator in predicting the clinical outcome of CAD patients (43). Concurrent studies confirmed that in SAP patients with T2DM, compared with the hemoglobin 
TABLE 4 | Estimated Kaplan-Meier events rates of 2.5 years follow-up.

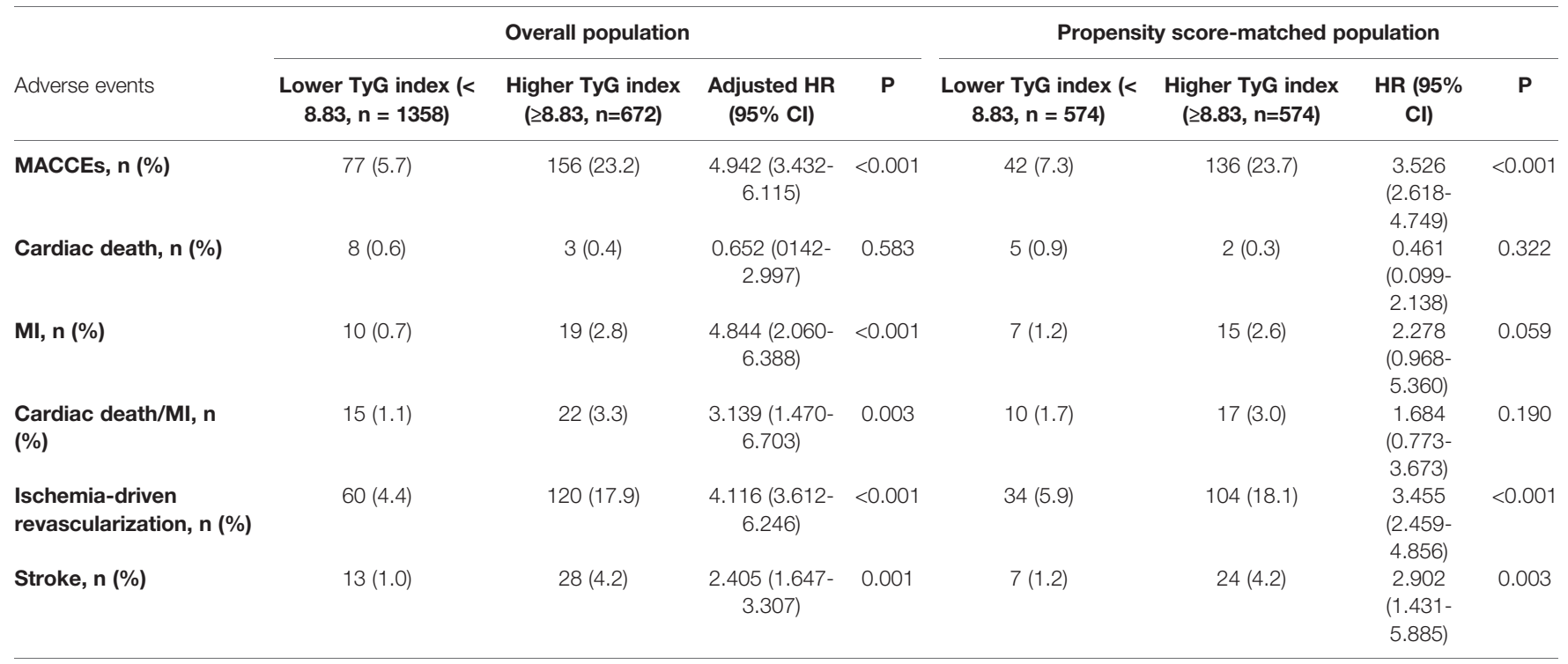

HR, hazard ratio; Cl, confidence level; TyG, triglyceride-glucose; MACCEs, major adverse cardiovascular and cerebrovascular events; MI, myocardial infarction.
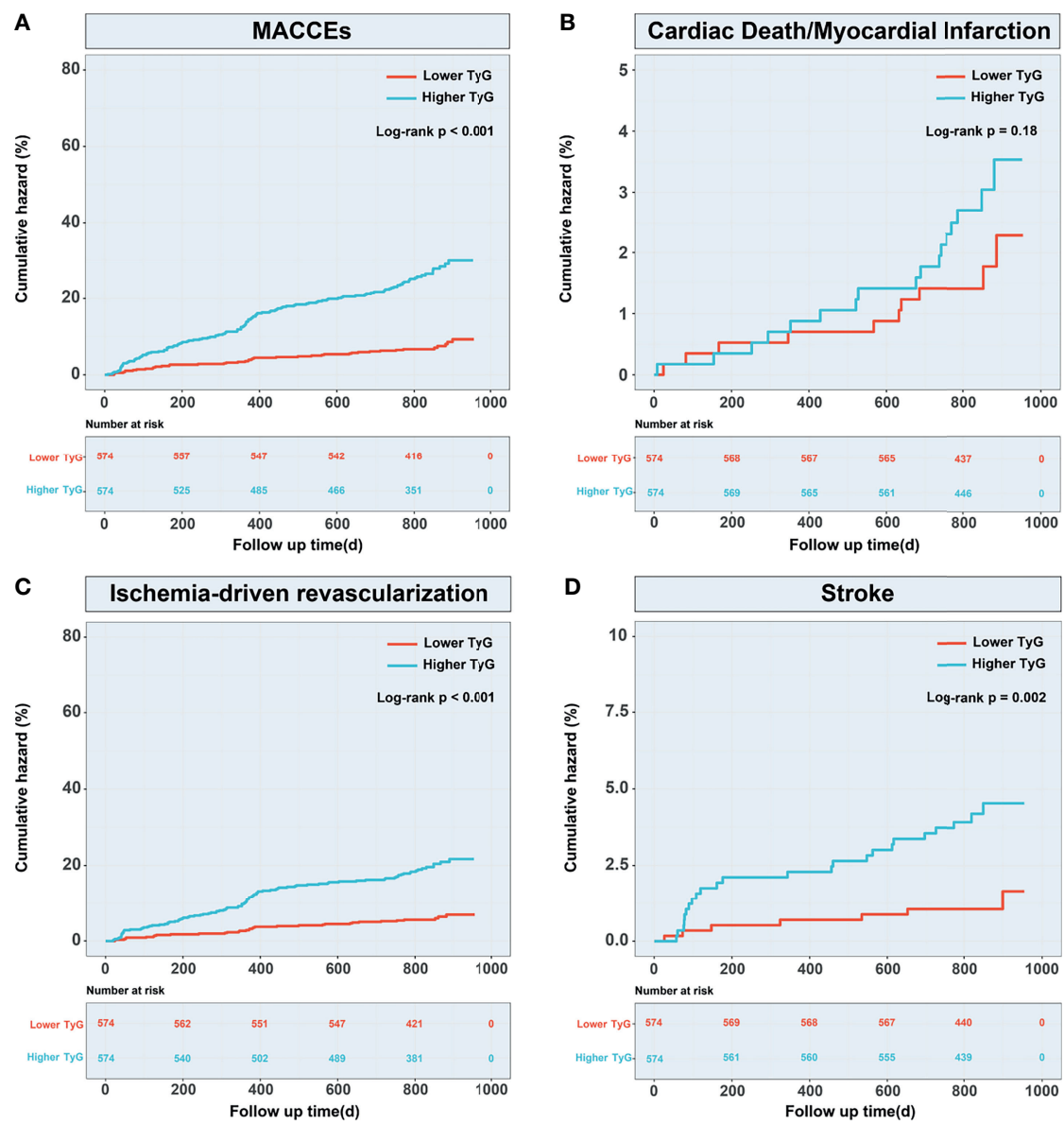

FIGURE 6 | Kaplan-Meier curves for MACCEs and endpoint events according to the propensity score-matched population. (A) Kaplan-Meier curves for MACCEs; (B) Kaplan-Meier curves for cardiac death or non-fatal myocardial infarction; (C) Kaplan-Meier curves for ischemia-driven revascularization; (D) Kaplan-Meier curves for stroke. TyG, triglyceride-glucose; MACCEs, major adverse cardiovascular and cerebrovascular events. 
glycation index, the TyG index is superior in terms of prognostic value (44).

In the ACS population, it has been found that high TyG indices may be related to a greater risk of major adverse cardiovascular events (MACE) in AMI patients (45). A recent study has shown that in NSTEMI patients, MACE occurred more frequently in patients with high TyG indices (46). In addition, the TyG index was also found to predict future MACE in patients with ACS (47).

Previous studies have shown that compared with $\mathrm{HbAlc}$ and TG, the TyG index is a valuable forecaster of future cardiovascular events, and may provide additional prognostic benefits for T2DM (48). For ACS patients treated with PCI, compared with FPG or $\mathrm{HbA1c}$, the TyG index may be superior in predicting cardiovascular events (49). In non-diabetic patients, a higher TyG index predicts future ischemic heart disease, indicating that it may be a valuable indicator for assessing the risk of cardiovascular events in nondiabetic adults (50). Similarly, in non-diabetic ACS patients, higher TyG indices are associated with a greater incidence of revascularization or AMI and larger infarct size (51), suggesting that high TyG indices can effectively predict revascularization. However, few studies have addressed the prognosis of prediabetic patients with ACS. The existing studies only report a causal relationship between prediabetes combined with all-cause mortality and CVD (29), with the TyG index recognized as potentially useful in the early detection of patients at risk of developing CVDs and adverse outcomes (52). In addition, prediabetes as a metabolic disorder might affect the MACCEs via over-inflammation and oxidative stress through inflammatory/ oxidative stress pathways at the levels of cardiac (fat) tissue and the atherosclerotic plaque $(12,13,53)$. The same pathways could be more evidenced in over-weight subjects and influence the epigenetic, the MACEs, and be influenced (positively) by metformin therapy (54). Moreover, prediabetic patients have alteration of inflammatory markers and of the value of endothelial function that consequently could cause higher rate of MACEs also in absence of significant coronary stenosis (55). In summary, our study indicates a close link between the TyG index and MACCEs in patients with prediabetes and ACS, with higher TyG indices related to poor prognosis. As the levels of the TyG index are rising, this may be a prognostic indicator for prediabetic patients with ACS.

\section{CONCLUSIONS}

The TyG index is an important simple composite index of IR in prediabetic patients, and high TyG indices may be significant prognostic indicators in prediabetic patients with ACS.

\section{LIMITATIONS}

(1) This is a single center study with a small sample size, and more multi-center studies need to be performed to confirm the present results. In addition, the retrospective nature of our study potentially biases the result of analysis. However, the prevalence of clinical risk factors in our study population was similar to some contemporary trials and real-world registries $(56,57)$. This might be a relevant finding and potentially supports the generalizability of our results. (2) TyG indices were only calculated at admission for only once and might change in the 2.5-year follow-up. However, we were not able to measure changes in TyG indices over time. (3) Due to lack of fasting insulin data, the calculation of HOMA-IR was not performed in the present study. (4) Since our study mainly focused on the presence of MACCEs in patients during the follow-up, we did not carry out subsequent hematological examination on these patients, and it was not possible to determine whether the patients progressed from prediabetes to diabetes or non-diabetes. (5) Outcome events in this study were not adjudicated by a clinical events committee. This represents a possible limitation of results that should be acknowledged (58).

\section{DATA AVAILABILITY STATEMENT}

The data supporting the conclusions of this study will be available from the corresponding authors on reasonable requests.

\section{ETHICS STATEMENT}

The studies involving human participants were reviewed and approved by the Institutional Ethics Committee of Beijing Anzhen Hospital. The data retrospectively obtained from electronic medical records.

\section{AUTHOR CONTRIBUTIONS}

QYG, XXF, BZ, and GYZ made contributions to the acquisition of data, analysis and drafting of the manuscript. QYG, XXF, YL, and JQY made contributions to the acquisition and interpretation of data. QYG, YJZ and DMS made substantial contributions to conception and design. YYL and YJZ made substantial contributions to critical revision of the manuscript. All authors read and approved the final manuscript.

\section{FUNDING}

This study was supported by the grant from Natural Science Foundation of Beijing, China (Grant No. 7214223) to QG. YZ was supported by National Key Research and Development Program of China (2017YFC0908800), Beijing Municipal Health Commission (Grant No. PXM2020_026272_000002 and Grant No. PXM2020_026272_000014) and Natural Science Foundation of Beijing, China (Grant No. 7212027).

\section{SUPPLEMENTARY MATERIAL}

The Supplementary Material for this article can be found online at: https://www.frontiersin.org/articles/10.3389/fendo.2022.843072/ full\#supplementary-material 


\section{REFERENCES}

1. Tabák AG, Herder C, Rathmann W, Brunner EJ, Kivimäki M. Prediabetes: A High-Risk State for Diabetes Development. Lancet (2012) 379(9833):2279-90. doi: 10.1016/S0140-6736(12)60283-9

2. Khetan AK, Rajagopalan S. Prediabetes. Can J Cardiol (2018) 34(5):615-23. doi: 10.1016/j.cjca.2017.12.030

3. Wilson ML. Prediabetes: Beyond the Borderline. Nurs Clin North Am (2017) 52(4):665-77. doi: 10.1016/j.cnur.2017.07.011

4. Salunkhe VA, Veluthakal R, Kahn SE, Thurmond DC. Novel Approaches to Restore Beta Cell Function in Prediabetes and Type 2 Diabetes. Diabetologia (2018) 61(9):1895-901. doi: 10.1007/s00125-018-4658-3

5. Huang Y, Cai X, Mai W, Li M, Hu Y. Association Between Prediabetes and Risk of Cardiovascular Disease and All Cause Mortality: Systematic Review and Meta-Analysis. BMJ (2016) 355:15953. doi: 10.1136/bmj.i5953

6. Mendez-Frausto G, Romero-Aguilera G, Sanchez-Gutierrez R, García-Jacobo RE, Lara-Ramírez EE, Uresti-Rivera EE, et al. B Regulatory Cells Associated With Changes in Biochemical and Inflammatory Parameters in NormalGlycemic Individuals, Pre-Diabetes and T2DM Patients. Diabetes Res Clin Pract (2021) 173:108692. doi: 10.1016/j.diabres.2021.108692

7. Vasques AC, Novaes FS, de Oliveira Mda S, Souza JR, Yamanaka A, Pareja JC, et al. TyG Index Performs Better Than HOMA in a Brazilian Population: A Hyperglycemic Clamp Validated Study. Diabetes Res Clin Pract (2011) 93(3): e98-100. doi: 10.1016/j.diabres.2011.05.030

8. Guerrero-Romero F, Villalobos-Molina R, Jiménez-Flores JR, SimentalMendia LE, Méndez-Cruz R, Murguía-Romero M, et al. Fasting Triglycerides and Glucose Index as a Diagnostic Test for Insulin Resistance in Young Adults. Arch Med Res (2016) 47(5):382-7. doi: 10.1016/ j.arcmed.2016.08.012

9. Stefan N, Fritsche A, Schick F, Häring HU. Phenotypes of Prediabetes and Stratification of Cardiometabolic Risk. Lancet Diabetes Endocrinol (2016) 4 (9):789-98. doi: 10.1016/S2213-8587(16)00082-6

10. Yang W, Lu J, Weng J, Jia W, Ji L, Xiao J, et al. Prevalence of Diabetes Among Men and Women in China. N Engl J Med (2010) 362(12):1090-101. doi: 10.1056/NEJMoa0908292

11. Li Y, Teng D, Shi X, Qin G, Qin Y, Quan H, et al. Prevalence of Diabetes Recorded in Mainland China Using 2018 Diagnostic Criteria From the American Diabetes Association: National Cross Sectional Study. BMJ (2020) 369:m997. doi: 10.1136/bmj.m997

12. Sardu C, D'Onofrio N, Torella M, Portoghese M, Mureddu S, Loreni F, et al. Metformin Therapy Effects on the Expression of Sodium-Glucose Cotransporter 2, Leptin, and SIRT6 Levels in Pericoronary Fat Excised From Pre-Diabetic Patients With Acute Myocardial Infarction. Biomedicines (2021) 9(8):904. doi: 10.3390/biomedicines 9080904

13. Sardu C, D'Onofrio N, Torella M, Portoghese M, Loreni F, Mureddu S, et al. Pericoronary Fat Inflammation and Major Adverse Cardiac Events (MACE) in Prediabetic Patients With Acute Myocardial Infarction: Effects of Metformin. Cardiovasc Diabetol (2019) 18(1):126. doi: 10.1186/s12933-0190931-0

14. Lee EY, Yang HK, Lee J, Kang B, Yang Y, Lee SH, et al. Triglyceride Glucose Index, a Marker of Insulin Resistance, Is Associated With Coronary Artery Stenosis in Asymptomatic Subjects With Type 2 Diabetes. Lipids Health Dis (2016) 15(1):155. doi: 10.1186/s12944-016-0324-2

15. da Silva A, Caldas APS, Rocha DMUP, Bressan J. Triglyceride-Glucose Index Predicts Independently Type 2 Diabetes Mellitus Risk: A Systematic Review and Meta-Analysis of Cohort Studies. Prim Care Diabetes (2020) 14(6):58493. doi: 10.1016/j.pcd.2020.09.001

16. Chamroonkiadtikun P, Ananchaisarp T, Wanichanon W. The TriglycerideGlucose Index, a Predictor of Type 2 Diabetes Development: A Retrospective Cohort Study. Prim Care Diabetes (2020) 14(2):161-7. doi: 10.1016/ j.pcd.2019.08.004

17. Wang Z, Zhao L, He S. Triglyceride-Glucose Index as Predictor for Future Type 2 Diabetes Mellitus in a Chinese Population in Southwest China: A 15Year Prospective Study. Endocrine (2021) 72(1):124-31. doi: 10.1007/s12020020-02589-7

18. Ramírez-Vélez R, Pérez-Sousa MÁ, González-Ruíz K, Cano-Gutierrez CA, Schmidt-RioValle J, Correa-Rodríguez M, et al. Obesity- and Lipid-Related Parameters in the Identification of Older Adults With a High Risk of
Prediabetes According to the American Diabetes Association: An Analysis of the 2015 Health, Well-Being, and Aging Study. Nutrients (2019) 11 (11):2654. doi: 10.3390/nu11112654

19. Sánchez-Íñigo L, Navarro-González D, Fernández-Montero A, PastranaDelgado J, Martínez JA. The TyG Index may Predict the Development of Cardiovascular Events. Eur J Clin Invest (2016) 46(2):189-97. doi: 10.1111/ eci. 12583

20. Heianza Y, Hara S, Arase Y, Saito K, Fujiwara K, Tsuji H, et al. HbAlc 5.76.4\% and Impaired Fasting Plasma Glucose for Diagnosis of Prediabetes and Risk of Progression to Diabetes in Japan (TOPICS 3): A Longitudinal Cohort Study. Lancet (2011) 378(9786):147-55. doi: 10.1016/S0140-6736(11)60472-8

21. American Diabetes Association. Classification and Diagnosis of Diabetes: Standards of Medical Care in Diabetes-2021. Diabetes Care (2021) 44(Suppl 1):S15-33. doi: $10.2337 / \mathrm{dc} 21-\mathrm{S} 002$

22. Collet JP, Thiele H, Barbato E, Barthélémy O, Bauersachs J, Bhatt DL, et al. ESC Guidelines for the Management of Acute Coronary Syndromes in Patients Presenting Without Persistent ST-Segment Elevation. Eur Heart J (2021) 42(14):1289-367. doi: 10.1093/eurheartj/ehaa575

23. Guerrero-Romero F, Simental-Mendía LE, González-Ortiz M, MartínezAbundis E, Ramos-Zavala MG, Hernández-González SO, et al. The Product of Triglycerides and Glucose, a Simple Measure of Insulin Sensitivity. Comparison With the Euglycemic-Hyperinsulinemic Clamp. J Clin Endocrinol Metab (2010) 95(7):3347-51. doi: 10.1210/jc.2010-0288

24. Kip KE, Hollabaugh K, Marroquin OC, Williams DO. The Problem With Composite End Points in Cardiovascular Studies: The Story of Major Adverse Cardiac Events and Percutaneous Coronary Intervention. J Am Coll Cardiol (2008) 51(7):701-7. doi: 10.1016/j.jacc.2007.10.034

25. Escaned J, Collet C, Ryan N, De Maria GL, Walsh S, Sabate M, et al. Clinical Outcomes of State-of-the-Art Percutaneous Coronary Revascularization in Patients With De Novo Three Vessel Disease: 1-Year Results of the SYNTAX II Study. Eur Heart J (2017) 38(42):3124-34. doi: 10.1093/eurheartj/ehx512

26. Kim MK, Ahn CW, Kang S, Nam JS, Kim KR, Park JS. Relationship Between the Triglyceride Glucose Index and Coronary Artery Calcification in Korean Adults. Cardiovasc Diabetol (2017) 16(1):108. doi: 10.1186/s12933-017-0589-4

27. Park K, Ahn CW, Lee SB, Kang S, Nam JS, Lee BK, et al. Elevated TyG Index Predicts Progression of Coronary Artery Calcification. Diabetes Care (2019) 42(8):1569-73. doi: 10.2337/dc18-1920

28. Bornfeldt KE, Tabas I. Insulin Resistance, Hyperglycemia, and Atherosclerosis. Cell Metab (2011) 14(5):575-85. doi: 10.1016/ j.cmet.2011.07.015

29. Kurihara O, Takano M, Yamamoto M, Shirakabe A, Kimata N, Inami T, et al. Impact of Prediabetic Status on Coronary Atherosclerosis: A Multivessel Angioscopic Study. Diabetes Care (2013) 36(3):729-33. doi: 10.2337/dc121635

30. Lee DY, Lee ES, Kim JH, Park SE, Park CY, Oh KW, et al. Predictive Value of Triglyceride Glucose Index for the Risk of Incident Diabetes: A 4-Year Retrospective Longitudinal Study. PloS One (2016) 11(9):e0163465. doi: 10.1371/journal.pone. 0163465

31. Nishikawa T, Edelstein D, Du XL, Yamagishi S, Matsumura T, Kaneda Y, et al. Normalizing Mitochondrial Superoxide Production Blocks Three Pathways of Hyperglycaemic Damage. Nature (2000) 404(6779):787-90. doi: 10.1038/ 35008121

32. Won KB, Chang HJ, Niinuma H, Sung J, Shin S, Cho IJ, et al. Evaluation of the Predictive Value of Coronary Artery Calcium Score for Obstructive Coronary Artery Disease in Asymptomatic Korean Patients With Type 2 Diabetes Mellitus. Coron Artery Dis (2015) 26(2):150-6. doi: 10.1097/MCA. 0000000000000184

33. Won KB, Kim YS, Lee BK, Heo R, Han D, Lee JH, et al. The Relationship of Insulin Resistance Estimated by Triglyceride Glucose Index and Coronary Plaque Characteristics. Med (Baltimore) (2018) 97(21):e10726. doi: 10.1097/ MD.0000000000010726

34. Zhou Y, Pan Y, Yan H, Wang Y, Li Z, Zhao X, et al. Triglyceride Glucose Index and Prognosis of Patients With Ischemic Stroke. Front Neurol (2020) 11:456:456. doi: 10.3389/fneur.2020.00456

35. Lee M, Kim CH, Kim Y, Jang MU, Mo HJ, Lee SH, et al. High Triglyceride Glucose Index Is Associated With Poor Outcomes in Ischemic Stroke Patients After Reperfusion Therapy. Cerebrovasc Dis (2021) 50(6):691-9. doi: 10.1159/ 000516950 
36. Zhang B, Liu L, Ruan H, Zhu Q, Yu D, Yang Y, et al. Triglyceride-Glucose Index Linked to Hospital Mortality in Critically Ill Stroke: An Observational Multicentre Study on eICU Database. Front Med (Lausanne) (2020) 7:591036. doi: 10.3389/fmed.2020.591036

37. Shi W, Xing L, Jing L, Tian Y, Yan H, Sun Q, et al. Value of TriglycerideGlucose Index for the Estimation of Ischemic Stroke Risk: Insights From a General Population. Nutr Metab Cardiovasc Dis (2020) 30(2):245-53. doi: 10.1016/j.numecd.2019.09.015

38. Zhao Y, Sun H, Zhang W, Xi Y, Shi X, Yang Y, et al. Elevated TriglycerideGlucose Index Predicts Risk of Incident Ischaemic Stroke: The Rural Chinese Cohort Study. Diabetes Metab (2021) 47(4):101246. doi: 10.1016/ j.diabet.2021.101246

39. Hou Z, Pan Y, Yang Y, Yang X, Xiang X, Wang Y, et al. An Analysis of the Potential Relationship of Triglyceride Glucose and Body Mass Index With Stroke Prognosis. Front Neurol (2021) 12:630140. doi: 10.3389/fneur. 2021.630140

40. Du Z, Xing L, Lin M, Sun Y. Estimate of Prevalent Ischemic Stroke From Triglyceride Glucose-Body Mass Index in the General Population. BMC Cardiovasc Disord (2020) 20(1):483. doi: 10.1186/s12872-020-01768-8

41. Luo E, Wang D, Yan G, Qiao Y, Liu B, Hou J, et al. High Triglyceride-Glucose Index Is Associated With Poor Prognosis in Patients With Acute ST-Elevation Myocardial Infarction After Percutaneous Coronary Intervention. Cardiovasc Diabetol (2019) 18(1):150. doi: 10.1186/s12933-019-0957-3

42. Li C, Liu Z. Bioinformatic Analysis for Potential Biomarkers and Therapeutic Targets of T2DM-Related MI. Int J Gen Med (2021) 14:4337-47. doi: 10.2147/ IJGM.S325980

43. Jin JL, Cao YX, Wu LG, You XD, Guo YL, Wu NQ, et al. Triglyceride Glucose Index for Predicting Cardiovascular Outcomes in Patients With Coronary Artery Disease. J Thorac Dis (2018) 10(11):6137-46. doi: 10.21037/jtd. 2018.10.79

44. Jin JL, Sun D, Cao YX, Guo YL, Wu NQ, Zhu CG, et al. Triglyceride Glucose and Haemoglobin Glycation Index for Predicting Outcomes in Diabetes Patients With New-Onset, Stable Coronary Artery Disease: A Nested CaseControl Study. Ann Med (2018) 50(7):576-86. doi: 10.1080/07853890. 2018.1523549

45. Zhang Y, Ding X, Hua B, Liu Q, Gao H, Chen H, et al. High TriglycerideGlucose Index Is Associated With Adverse Cardiovascular Outcomes in Patients With Acute Myocardial Infarction. Nutr Metab Cardiovasc Dis (2020) 30(12):2351-62. doi: 10.1016/j.numecd.2020.07.041

46. Mao Q, Zhou D, Li Y, Wang Y, Xu SC, Zhao XH. The Triglyceride-Glucose Index Predicts Coronary Artery Disease Severity and Cardiovascular Outcomes in Patients With Non-ST-Segment Elevation Acute Coronary Syndrome. Dis Markers (2019) 2019:6891537. doi: 10.1155/2019/6891537

47. Li J, Ren L, Chang C, Luo L. Triglyceride-Glukose Index Predicts Adverse Events in Patients With Acute Coronary Syndrome: A Meta-Analysis of Cohort Studies. Horm Metab Res (2021) 53(9):594-601. doi: 10.1055/a-15187503

48. Su WY, Chen SC, Huang YT, Huang JC, Wu PY, Hsu WH, et al. Comparison of the Effects of Fasting Glucose, Hemoglobin A1c, and Triglyceride-Glucose Index on Cardiovascular Events in Type 2 Diabetes Mellitus. Nutrients (2019) 11(11):2838. doi: 10.3390/nu11112838

49. Cui J, Liu Y, Li Y, Xu F, Liu Y. Type 2 Diabetes and Myocardial Infarction: Recent Clinical Evidence and Perspective. Front Cardiovasc Med (2021) 8:644189. doi: 10.3389/fcvm.2021.644189

50. Yang J, Tang YD, Zheng Y, Li C, Zhou Q, Gao J, et al. The Impact of the Triglyceride-Glucose Index on Poor Prognosis in NonDiabetic Patients
Undergoing Percutaneous Coronary Intervention. Front Endocrinol (Lausanne) (2021) 12:710240. doi: 10.3389/fendo.2021.710240

51. Zhang Y, Ding X, Hua B, Liu Q, Gao H, Chen H, et al. High TriglycerideGlucose Index Is Associated With Poor Cardiovascular Outcomes in Nondiabetic Patients With ACS With LDL-C Below $1.8 \mathrm{Mmol} / \mathrm{L}$. J Atheroscler Thromb (2022) 29(2):268-81. doi: 10.5551/jat.61119

52. Hong S, Han K, Park CY. The Triglyceride Glucose Index Is a Simple and Low-Cost Marker Associated With Atherosclerotic Cardiovascular Disease: A Population-Based Study. BMC Med (2020) 18(1):361. doi: 10.1186/s12916020-01824-2

53. Sardu C, Modugno P, Castellano G, Scisciola L, Barbieri M, Petrella L, et al. Atherosclerotic Plaque Fissuration and Clinical Outcomes in Pre-Diabetics vs. Normoglycemics Patients Affected by Asymptomatic Significant Carotid Artery Stenosis at 2 Years of Follow-Up: Role of microRNAs Modulation: The ATIMIR Study. Biomedicines (2021) 9(4):401. doi: 10.3390/ biomedicines 9040401

54. Sardu C, Trotta MC, Pieretti G, Gatta G, Ferraro G, Nicoletti GF, et al. MicroRNAs Modulation and Clinical Outcomes at 1 Year of Follow-Up in Obese Patients With Pre-Diabetes Treated With Metformin vs. Placebo. Acta Diabetol (2021) 58(10):1381-93. doi: 10.1007/s00592-021-01743-5

55. Sardu C, Paolisso P, Sacra C, Mauro C, Minicucci F, Portoghese M, et al. Effects of Metformin Therapy on Coronary Endothelial Dysfunction in Patients With Prediabetes With Stable Angina and Nonobstructive Coronary Artery Stenosis: The CODYCE Multicenter Prospective Study. Diabetes Care (2019) 42(10):1946-55. doi: 10.2337/dc18-2356

56. Valgimigli M, Gragnano F, Branca M, Franzone A, Baber U, Jang Y, et al. P2Y12 Inhibitor Monotherapy or Dual Antiplatelet Therapy After Coronary Revascularisation: Individual Patient Level Meta-Analysis of Randomised Controlled Trials. BMJ (2021) 373:n1332. doi: 10.1136/bmj.n1332

57. Cesaro A, Gragnano F, Calabrò P, Moscarella E, Santelli F, Fimiani F, et al. Prevalence and Clinical Implications of Eligibility Criteria for Prolonged Dual Antithrombotic Therapy in Patients With PEGASUS and COMPASS Phenotypes: Insights From the START-ANTIPLATELET Registry. Int J Cardiol (2021) 345:7-13. doi: 10.1016/j.ijcard.2021.10.138

58. Leonardi S, Branca M, Franzone A, McFadden E, Piccolo R, Jüni P, et al. Comparison of Investigator-Reported and Clinical Event CommitteeAdjudicated Outcome Events in GLASSY. Circ Cardiovasc Qual Outcomes (2021) 14(2):e006581. doi: 10.1161/CIRCOUTCOMES.120.006581

Conflict of Interest: The authors declare that the research was conducted in the absence of any commercial or financial relationships that could be construed as a potential conflict of interest.

Publisher's Note: All claims expressed in this article are solely those of the authors and do not necessarily represent those of their affiliated organizations, or those of the publisher, the editors and the reviewers. Any product that may be evaluated in this article, or claim that may be made by its manufacturer, is not guaranteed or endorsed by the publisher.

Copyright (c) 2022 Guo, Feng, Zhang, Zhai, Yang, Liu, Liu, Shi and Zhou. This is an open-access article distributed under the terms of the Creative Commons Attribution License (CC BY). The use, distribution or reproduction in other forums is permitted, provided the original author(s) and the copyright owner(s) are credited and that the original publication in this journal is cited, in accordance with accepted academic practice. No use, distribution or reproduction is permitted which does not comply with these terms. 